\title{
Addition Reaction of Phenylphosphines to Ethynylbenzenes as the Model of Polyaddition. Study on the Rate-Determining Step and Substituent Effect
}

\author{
Eiichi Kobayashi*, Takatsugu Obata, Sadahito Aoshima, \\ and Junji Furukawa \\ Department of Industrial Chemistry, Faculty of Science and Technology, \\ Science University of Tokyo, Noda, Chiba 278, Japan
}

(Received February 17, 1993)

\begin{abstract}
To know the behavior and mechanism of the radical addition reactions of phosphine compounds to ethynyl compounds, model addition reactions were investigated in detail. In the addition reaction of phenylphosphine to ethynylbenzene, phenylphosphine having two reactive hydrogens added to two ethynylbenzenes by the anti-Markownikoff's rule to give the $1: 2$ adduct $[\phi \mathrm{CH}=\mathrm{CHP}(\phi) \mathrm{CH}=\mathrm{CH} \phi]$ in a high yield. The reactivity of the first addition of phenylphosphine to ethynylbenzene was lower than that of the second addition of $\phi \mathrm{CH}=\mathrm{CHPH} \phi$ to ethynylbenzene. This result differs from the case of the addition reaction to styrene. The kinetic investigation was also carried out using the addition reaction of diphenylphosphine to ethynylbenzene, to give the $1: 1$ adduct in a high yield. The rate-determining step was the chain transfer reaction between the intermediate carbon radical $\left(\phi \dot{\mathrm{C}}=\mathrm{CHP} \phi_{2}\right)$ and diphenylphosphine. The substituent effect of the addition reactions of diphenylphosphine to $p$-substituted ethynylbenzenes was studied by modified Hammett's equation. The results were compared with those of the addition reactions of diphenylphosphine to styrenes. Based on the results of these model reactions the polyaddition behavior of phenylphosphine to 1,4-diethynylbenzene and the polymer structure were discussed.

KEY WORDS Kinetics / Polyaddition / Addition Reaction / Reaction

Mechanism / Rate-Determining Step / Substituent Effect / Hammett

Correlation / Ethynylbenzene Derivatives / Phenylphosphine / Diphenylphosphine /
\end{abstract}

Phosphines have been reported to add to ethynyl compounds as well as to olefins in the presence of a radical initiator, but the yields of the adducts were relatively low. For example, bis(2-cyanoethyl)phosphine reacted with 1heptyne or with 1-octyne in the presence of AIBN to give the unsaturated $1: 1$ phosphine adducts in $27-30 \%$ yields. ${ }^{1}$ In the case of primary phosphine, the addition reaction of 2-cyanoethylphosphine to 1-heptyne took place and the $1: 2$ adduct of the phosphine and 1-heptyne was obtained in a $26 \%$ yield. ${ }^{1}$ The
$1: 1$ adduct having the reactive $\mathrm{P}-\mathrm{H}$ might be more reactive than the starting primary phosphine. On the other hand, the radical cycloaddition of phenylphosphine to hexa-1,5diyne was also carried out to obtain 4,5dihydrophosphepin by Maerkl et al. ${ }^{2}$ However, no extensive investigations such as reaction mechanism and substituent effect have been done.

In the present paper, to study the behavior of the radical addition reactions of phosphine compounds to ethynyl compounds, the model

\footnotetext{
* To whom all correspondence should be addressed.
} 
addition reaction of phenylphosphine $(\mathrm{PH})$ to ethynylbenzene (EB) was investigated in detail. The kinetic investigation of the addition reaction of diphenylphosphine (DPH) to EBs was also examined, and the reaction mechanism and substituent effect were discussed. Furthermore, the polyaddition behavior of $\mathrm{PH}$ to 1,4-diethynylbenzene (DEB) and the obtained polymer structure were discussed from these model reactions.

\section{EXPERIMENTAL}

\section{Reagents}

$\mathrm{PH},{ }^{3} \mathrm{DPH},{ }^{4}$ and three EBs ( $p$-methoxy, ${ }^{5}$ $p$-methyl, ${ }^{5}$ and $p$-chloro $\left.{ }^{6} \mathrm{EBs}\right)$ were synthesized according to the literatures. EB (Wako Pure Chemical Industries, Ltd.) was obtained commercially. The reagents were purified more than twice by vacuum distillation under a nitrogen atmosphere before use. Toluene (Nacalai Tesque Inc.) as a reaction solvent was distilled over $\mathrm{CaH}_{2}$ and then deairing to exclude oxygen by vacuum for $20 \mathrm{~min}$ before use. 2,2'-Azobisisobutyronitrile (AIBN, Wako Pure Chemical Industries, Ltd.) was used as received. Four nine nitrogen gas was purified as shown in the previous paper. ${ }^{7}$

\section{Addition Reaction}

The addition reaction of $\mathrm{PH}$ to $\mathrm{EB}$ was carried out in a sealed pyrex tube by mixing PH $\left(0.5 \mathrm{moll}^{-1}\right)$ and EB $\left(1.0 \mathrm{moll}^{-1}\right)$ in the presence of AIBN $\left(0.04 \mathrm{moll}^{-1}\right)$ at $60^{\circ} \mathrm{C}$ in toluene under a nitrogen atmosphere. After the prescribed time, the sealed tube was opened, and the concentrations of $\mathrm{PH}, \mathrm{EB}$, and the products were determined by GC using decane as an internal reference.

In the case of the kinetic investigation, the reaction was carried out in a $50 \mathrm{ml}$ roundbottomed flask equipped with a three-way stopcock by introducing the toluene solutions of DPH, EBs, AIBN, and cis-decalin or decane as an internal reference using a hypodermic syringe under a nitrogen atmosphere. The reaction temperature was maintained at $60^{\circ} \mathrm{C}$. The reaction course was monitored every $c a$. $10 \mathrm{~min}$ by measurement of the concentration of DPH by GC.

\section{Measurements}

Gas chromatograms were measured on a Shimadzu GC-4BMPF equipped with a flame ionization detector (FID) and a Silicone GE SE-30 column $(2 \mathrm{~m})$ under a nitrogen carrier gas at $300^{\circ} \mathrm{C}$ of injection temperature. The GC conditions were same as the addition reaction of PH or DPH to Sts. ${ }^{8}{ }^{1} \mathrm{H}$ NMR and IR spectra were recorded on a JEOL JNM-EX400 in $\mathrm{CDCl}_{3}$ with TMS standard and on a Hitachi 260-50 spectrophotometer by a $\mathrm{KBr}$ method, respectively. The molecular weight distributions of the products were measured in chloroform on a JASCO TRI ROTAR gel permeation chromatography (GPC) equipped with a polystyrene gel column (Shodex K-2002) and a refractive index detector. FD mass spectrum was obtained on a JEOL JMSMX110. Phosphorus content of the adducts was determined by the phosphovanadomolybdic acid method ${ }^{9,10}$ as described in the previous paper. ${ }^{11}$

\section{RESULTS AND DISCUSSION}

\section{Addition Reaction of $\mathrm{PH}$ to $\mathrm{EB}$}

In the previous work, ${ }^{8}$ it was found that $\mathrm{PH}$ having two hydrogens added to two styrenes (St) to give the $1: 2$ adduct of the antiMarkownikoff's type in a high yield. Some side reactions occurred and the information with these model reactions was utilized for the preparation of soluble phosphine polymers of PH and DVB. ${ }^{11}$ In this paper, thus, to know the polyaddition behavior of PH to DEB and the polymer structure, the model addition reaction of $\mathrm{PH}$ to $\mathrm{EB}$ was investigated in detail.

The addition reaction of $\mathrm{PH}\left(0.5 \mathrm{moll}^{-1}\right)$ to EB $\left(1.0 \mathrm{moll}^{-1}\right)$ was carried out with AIBN initiator in toluene at $60^{\circ} \mathrm{C}$ under a nitrogen atmosphere. Since $\mathrm{PH}$ contains two $\mathrm{P}-\mathrm{H}$ 


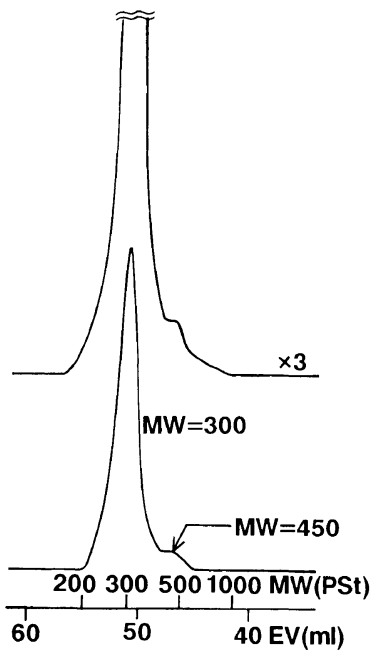

Figure 1. Molecular weight distribution curve of the products obtained for addition reaction of $\mathrm{PH}$ to $\mathrm{EB}$ for 2 days at $60^{\circ} \mathrm{C}$ in toluene under a nitrogen atmosphere. $[\mathrm{PH}]_{0}=0.5 \mathrm{moll}^{-1} ;[\mathrm{EB}]_{0}=1.0 \mathrm{moll}^{-1} ;[\mathrm{AIBN}]_{0}=0.04$ moll ${ }^{-1}$.
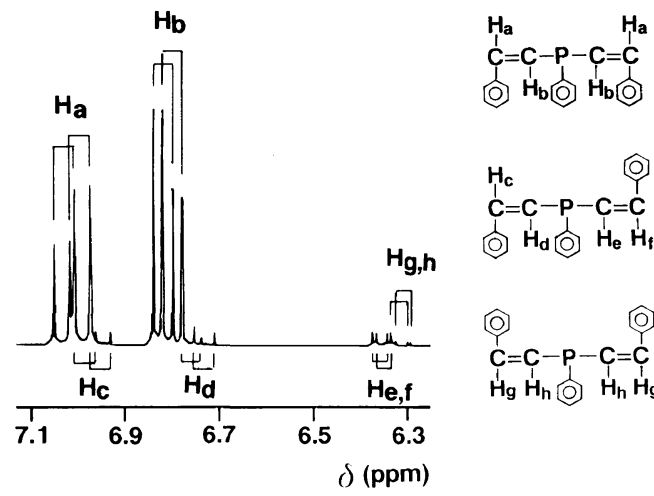

Figure 2. ${ }^{1} \mathrm{H}$ NMR spectrum of the isolated adduct of $\mathrm{PH}$ and $\mathrm{EB}$ in $\mathrm{CDCl}_{3}$.

bonds, twice molar quantity of EB to $\mathrm{PH}$ was added. The addition reaction completed for 2 days, and the adduct was obtained in a $97 \%$ yield. Figure 1 shows the molecular weight distribution curve of the products.

The main adduct isolated by vacuum distillation (bp $146^{\circ} \mathrm{C} / 0.01 \mathrm{mmHg}$ ) was a pale yellow solid. The ${ }^{1} \mathrm{H}$ NMR spectrum of the isolated adduct shows signals at $\delta 6.71-7.05$ ppm due to trans vinylene protons $[\delta(\mathrm{ppm})$;
$7.02\left(\mathrm{H}_{\mathrm{a}}\right), 6.81\left(\mathrm{H}_{\mathrm{b}}\right), 6.97\left(\mathrm{H}_{\mathrm{c}}\right), 6.75\left(\mathrm{H}_{\mathrm{d}}\right)$; $J_{\mathrm{ab}}=J_{\mathrm{cd}}=17.1 \mathrm{~Hz} ;{ }^{3} J_{\mathrm{PC}=\mathrm{CH}_{\mathrm{a}}}=13.7 \mathrm{~Hz},{ }^{2} J_{\mathrm{PCH}_{\mathrm{b}}}=$ $\left.7.8 \mathrm{~Hz},{ }^{3} J_{\mathrm{PC}=\mathrm{CH}_{\mathrm{c}}}=12.7 \mathrm{~Hz},{ }^{2} J_{\mathrm{PCH}_{\mathrm{d}}}=10.7 \mathrm{~Hz}\right]$, $6.30-6.38 \mathrm{ppm}$ due to $\mathrm{cis}$ vinylene protons $[\delta$ $(\mathrm{ppm}) ; 6.35\left(\mathrm{H}_{\mathrm{e}, \mathrm{f}}\right), 6.32\left(\mathrm{H}_{\mathrm{g}, \mathrm{h}}\right) ; J_{\mathrm{ef}}=J_{\mathrm{gh}}=$ $12.7 \mathrm{~Hz}]$, and at $\delta 7.22-7.56 \mathrm{ppm}$ due to benzene ring protons as shown in Figure 2. Since the assignment of the spectrum is tentative, the exact assignment will be confirmed by the newly synthesized authentic samples having pure cis or trans form. The spectrum also indicates the absence of the signals of unreacted $\mathrm{P}-\mathrm{H}$ group or the signals indicative of the side reactions such as Markownikoff's type reactions. On the basis of the phosphorus content of the adduct (found $10.0 \mathrm{wt} \%$; calcd $9.9 \mathrm{wt} \%$ ) and the ${ }^{1} \mathrm{H}$ NMR spectrum, it was deduced that both hydrogens in $\mathrm{PH}$ could be reacted with $\mathrm{EB}$ by the anti-Markownikoff's rule to give the $1: 2$ adduct 2 of PH and EB as shown in eq 1.

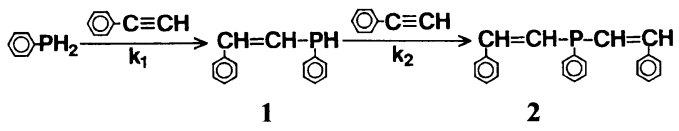

The gas chromatogram of the isolated adduct shows the three peaks at the retention times of $13.9,17.5$, and $21.1 \mathrm{~min}$ (flow rate of nitrogen carrier gas, $35 \mathrm{ml} \mathrm{min}^{-1}$; column temp, $270^{\circ} \mathrm{C}$; injection temp, $300^{\circ} \mathrm{C}$ ). These peaks were assigned by GC and ${ }^{1} \mathrm{H}$ NMR analyses to be the cis-cis, cis-trans, and trans-trans isomers of the $1: 2$ adduct 2 , respectively. The adduct in the reaction mixture was confirmed to have the $52 \%$ cis-cis, $30 \%$ cis-trans, and $18 \%$ trans-trans structures by GC analysis, which was consistent with that of the polymer of $\mathrm{PH}$ and DEB (cis/trans $\simeq 55 / 45$ ). But after distillation the content in the isolated adduct changed to $5 \%$ cis-cis, $22 \%$ cis-trans, and $73 \%$ trans-trans structures. That is, the adduct was found to be isomerized from cis to trans structures by the heat mode. The detailed isomerization behavior of the adducts will be 
reported in the next paper.

The FD mass spectrum of the reaction mixture shows the molecular ion peaks at $\mathrm{m} / \mathrm{z}$ 314 [MW, 300 (GPC); 314 (calcd)] due to the $1: 2$ adduct 2 , and at $m / z 416$ and 524 due to by-products. The by-product with MW 416 is the $1: 3$ adduct [MW, 450 (GPC); 416 (calcd)] of $\mathrm{PH}$ and EB. But, the by-product with MW 524 is unclear, and may be cyclic or dehydrogenated compound of $2: 3$ adduct [no peak by GPC; MW, 524 (calcd)] of PH and EB.

To know the ratio of the apparent second addition rate constant $\left(k_{2}\right)$ to the apparent first addition rate constant $\left(k_{1}\right), k_{2} / k_{1}$, the concentrations of both remaining monomers of $\mathrm{PH}$ and EB, and the yields of the products were monitored by GC. As shown in Figure 3, both monomers decreased and the yield of the adduct 2 increased with reaction time, respectively, whereas the $1: 1$ adduct 1 of $\mathrm{PH}$ and EB was not detected. That is, the reactivity of the first addition of $\mathrm{PH}$ to EB is lower than that of the second addition of $\phi \mathrm{CH}=\mathrm{CHPH} \phi$ to EB: $k_{1} \ll k_{2}$, while the reactivity of the first addition of $\mathrm{PH}$ to $\mathrm{St}$ was higher than that of the second addition, ${ }^{8} k_{1}>k_{2}$. That is, the hydrogen abstraction from 1 is probably to occur more rapidly than that from $\mathrm{PH}$, because $\phi \mathrm{CH}=\mathrm{CH} \dot{\mathrm{P}} \phi$ formed from 1 has a larger resonance stability than $\phi \dot{\mathrm{P}} \mathrm{H}$ formed from $\mathrm{PH}$.

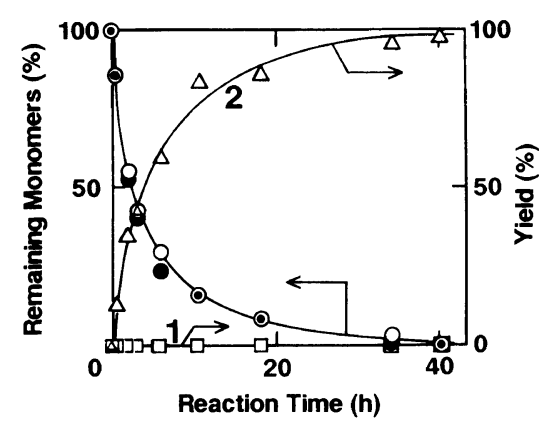

Figure 3. Time-courses of the remaining monomers or the yields of $\mathbf{1}$ and $\mathbf{2}$ for addition reaction of $\mathrm{PH}$ to EB at $60^{\circ} \mathrm{C}$ in toluene under a nitrogen atmosphere. $[\mathrm{PH}]_{0}=0.5$ $\mathrm{moll}^{-1} ;[\mathrm{EB}]_{0}=1.0 \mathrm{moll}^{-1} ;[\mathrm{AIBN}]_{0}=0.04 \mathrm{moll}^{-1}$; int. ref. for GC, decane. $\mathrm{O}, \mathrm{PH} ; \boldsymbol{O}, \mathrm{EB} ; \square, \phi \mathrm{CH}=\mathrm{CHPH} \phi$ (1); $\triangle, \phi \mathrm{CH}=\mathrm{CHP}(\phi) \mathrm{CH}=\mathrm{CH} \phi(2)$.
The authors propose the polyaddition behavior of PH to DEB as follows: the polymer might have an alternating structure of $\mathrm{PH}$ and DEB. However, a minor homosequence of DEB-DEB unit is probably formed in the polymer main chain, which is suggested by the formation of $1: 3$ adduct of $\mathrm{PH}$ and EB in the model addition reaction. The pendant ethynyl group thus formed induces polymer crosslinkage. The polymer of a molecular weight of about $8 \times 10^{3}$ may have potentially only one pendant ethynyl group according to the calculation based on the by-product content in the model addition reaction of $\mathrm{PH}$ to $\mathrm{EB}$. Therefore, the polymers with the molecular weight of lower than $8 \times 10^{3}$ are expected to be soluble in conventional organic solvents. In fact, the polyaddition of PH to DEB occurred by the anti-Markownikoff's rule, and the obtained polymer $\left(\bar{M}_{w}=5.2 \times 10^{3}\right)$ was soluble in chloroform, THF, and DMF etc. in spite of the conjugated polymer structure. ${ }^{12}$

\section{Addition Reaction of DPH to EB}

The kinetic study of the radical addition reactions of phosphine compounds to ethynyl compounds may be helpful to know the reaction mechanism, and to synthesize new phosphine compounds and polymers. In this section, to simplify the addition reaction, the kinetic investigation was achieved using DPH having mono reactive hydrogen, instead of $\mathrm{PH}$. The addition reaction of DPH $\left(1.0 \mathrm{moll}^{-1}\right)$ to EB $\left(1.0 \mathrm{moll}^{-1}\right)$ was carried out with AIBN initiator $\left(0.04 \mathrm{moll}^{-1}\right)$ at $60^{\circ} \mathrm{C}$ in toluene under a nitrogen atmosphere. The reaction was completed for 2 days without appreciable side reactions, and the adduct of the antiMarkownikoff's structure was obtained in a 97\% yield (Table I in the latter section). The relative addition reactivity of DPH to EB is found to be almost equal to that of $\mathrm{PH}$ to EB, which is confirmed by the competitive reaction of DPH and PH to EB (Figure 4). Accordingly, the addition reaction of DPH to EB is suggested to proceed by a similar mechanism 


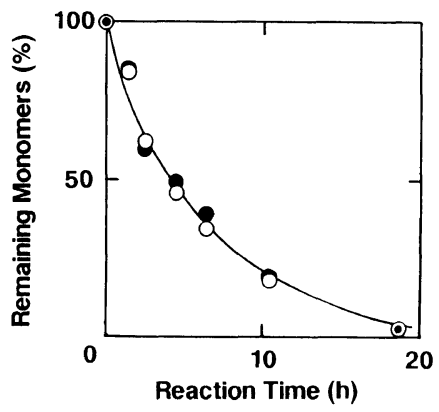

Figure 4. Competitive addition reaction of DPH $(\bullet)$ and $\mathrm{PH}(\mathrm{O})$ to $\mathrm{EB}$ at $60^{\circ} \mathrm{C}$ in toluene under a nitrogen atmosphere. $[\mathrm{DPH}]_{0}=[\mathrm{PH}]_{0}=0.5 \mathrm{moll}^{-1} ;[\mathrm{EB}]_{0}=2.5$ $\mathrm{moll}^{-1} ;[\mathrm{AIBN}]_{0}=0.04 \mathrm{moll}^{-1}$.

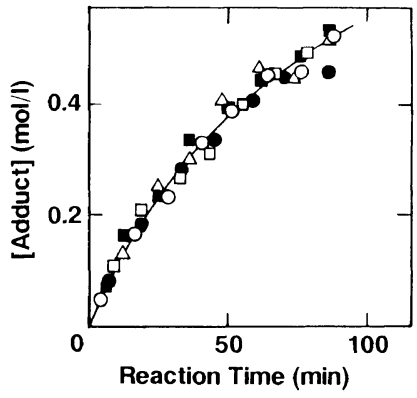

Figure 5. Time-yield curves of the adduct formation of DPH to EB by changing the initial concentration of EB at $60^{\circ} \mathrm{C}$ in toluene under a nitrogen atmosphere. $[\mathrm{DPH}]_{0}=1.04 \mathrm{moll}^{-1} ;[\mathrm{AIBN}]_{0}=0.04 \mathrm{moll}^{-1}$; int. ref. for $\mathrm{GC}$, cis-decalin. $[\mathrm{EB}]_{0}\left(\mathrm{mol1}^{-1}\right): \square, 2.03 ; \triangle, 1.56 ; \bigcirc$, $1.03 ; \square, 0.71 ; \bigcirc, 0.51$.

to the addition reaction of $\mathrm{PH}$ to $\mathrm{EB}$. The addition reaction of DPH to EB can be utilized for the kinetic investigation as a model of polyaddition of $\mathrm{PH}$ to DEB.

Reaction Order and Rate-Determining Step. The reaction order of the addition reaction of DPH to EB was estimated by the similar method to that described in the previous paper. ${ }^{7,13}$ As shown in Figure 5 the rate of addition reaction was found to be independent of the EB concentration. On the other hand, the typical time-yield curves and the reaction order of DPH in Figures 6(a) and 6(b), respectively, indicate that the addition rate depends on the first order of the DPH concentration. The kinetic order of AIBN was
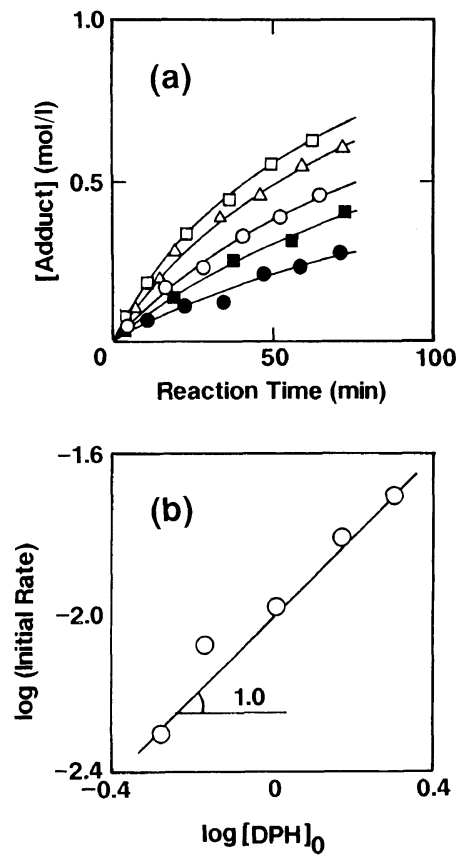

Figure 6. Time-yield curves (a) of the adduct formation of DPH to EB by changing the initial concentration of $\mathrm{DPH}$ and the reaction order (b) of DPH at $60^{\circ} \mathrm{C}$ in toluene under a nitrogen atmosphere. $[\mathrm{EB}]_{0}=1.03 \mathrm{moll}^{-1}$; $[\mathrm{AIBN}]_{0}=0.04 \mathrm{moll}^{-1}$; int. ref. for $\mathrm{GC}$, cis-decalin. $[\mathrm{DPH}]_{0}\left(\mathrm{moll}^{-1}\right): \square, 2.05 ; \triangle, 1.52 ; \bigcirc, 1.04 ; \square, 0.68$; 0.53 .

determined to be approximately 0.5 , indicating that the addition reaction progresses by the radical chain mechanism. Thus, the reaction rate is expressed in eq 2 ;

$$
\text { Rate }=k[\mathrm{~EB}]^{0}[\mathrm{DPH}]^{0}[\mathrm{AIBN}]^{0.5}
$$

where $k$ is overall reaction rate constant in toluene at $60^{\circ} \mathrm{C}$. By the theoretical rate equation, ${ }^{13}$ the results indicate that the rate-determining step is the chain transfer step between the intermediate carbon radical $(\dot{\mathrm{C}}$ : $\left.\phi \dot{\mathrm{C}}=\mathrm{CHP} \phi_{2}\right)$ and DPH.

Substituent Effect. The substituent effect on the addition reactions of DPH to $p$-substituted EBs, such as $p$-methoxy, $p$-methyl, and $p$ chloro EBs, was also investigated. All addition reactions of DPH to EBs were studied under the similar conditions to those of DPH to EB, and the adducts of the anti-Markow- 
E. Kobayashi et al.

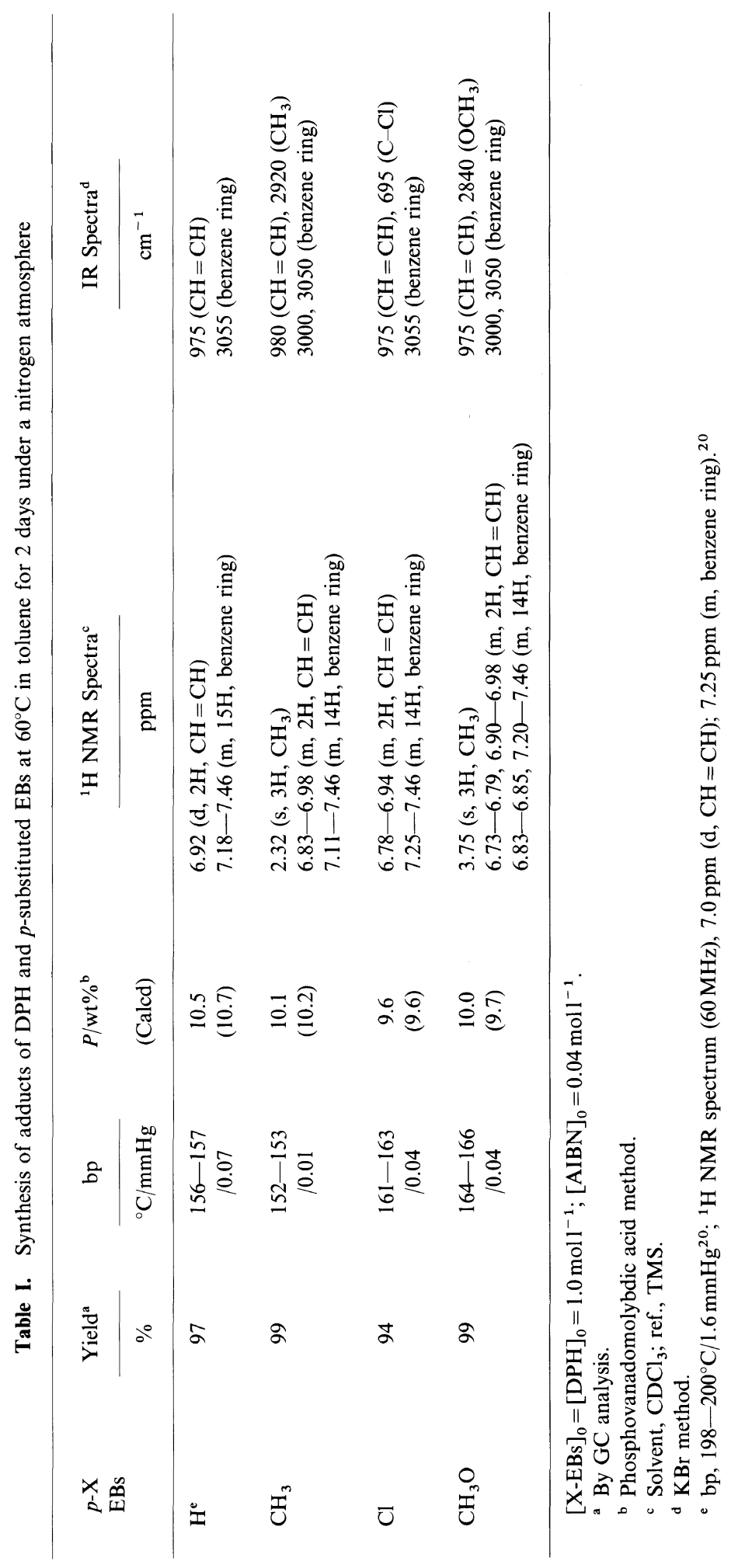


Table II. Relative rates $\left(k_{\mathrm{tr}}^{\mathbf{X}} / k_{\mathrm{tr}}^{\mathrm{H}}\right)^{\mathrm{a}}$ of the chain transfer step in the addition reactions of DPH to $p$-X EBs at $60^{\circ} \mathrm{C}$ in toluene under a nitrogen atmosphere

\begin{tabular}{lc}
\hline$p-\mathrm{X}$ & $k_{\mathrm{tr}}^{\mathrm{X}} / K_{\mathrm{tr}}^{\mathrm{H}}$ \\
$\mathrm{EBs}$ & 1.28 \\
$\mathrm{MeO}$ & 1.38 \\
$\mathrm{Me}$ & 1.00 \\
$\mathrm{H}$ & 0.32 \\
$\mathrm{Cl}$ &
\end{tabular}

${ }^{\mathrm{a}}[\mathrm{X}-\mathrm{EBs}]_{0}=[\mathrm{DPH}]_{0}=1.0 \mathrm{moll}^{-1} ; \quad[\mathrm{AIBN}]_{0}=0.04$ moll $^{-1}$.

nikoff's structure were obtained in $94-99 \%$ yields as shown in Table I. The relative rates, $k_{\mathrm{tr}}^{\mathbf{X}} / k_{\mathrm{tr}}^{\mathbf{H}}$, of the chain transfer step, which is the rate-determining step, were determined by the similar method to the thiobenzoic acid- $-\mathrm{X}$ EBs systems having the same rate-determining step. ${ }^{14}$ The $k_{\mathrm{tr}}^{\mathrm{X}}$ or $k_{\mathrm{tr}}^{\mathrm{H}}$ is the chain transfer rate constant between the $p$-X or $p-\mathrm{H}$ intermediate carbon radical and $\mathrm{DPH}$, respectively. The relative rates, $k_{\mathrm{tr}}^{\mathbf{X}} / k_{\mathrm{tr}}^{\mathbf{H}}$, are summarized in Table II, and the plots by Hammett's equation 3 (broken line) and modified Hammett's equation $4^{15}$ (solid line) are shown in Figure 7.

$$
\begin{gathered}
\log \left(k_{\mathrm{tr}}^{\mathbf{X}} / k_{\mathrm{tr}}^{\mathbf{H}}\right)=\rho \sigma \\
\log \left(k_{\mathrm{tr}}^{\mathbf{X}} / k_{\mathrm{tr}}^{\mathbf{H}}\right)=\rho \sigma+\gamma E_{\mathrm{R}}
\end{gathered}
$$

Better correlation was obtained with eq 4 , indicating that the chain transfer rates are controlled by both polar and resonance effects. Both the effects were already discussed with the model addition reactions of thiophenols and thiobenzoic acids to Sts and EBs. ${ }^{7,14,16}$

The reaction constant, $\rho$, obtained tentatively from Figure 7 was negative value, -1.26 . Imoto ${ }^{17}$ discussed a magnitude of the electron transfer between reactive molecules in the transition state by the $\rho$ value. That is, the electron is somewhat transferred from the intermediate carbon radical $\dot{\mathbf{C}}$ to $\mathrm{DPH}$ in the transition state. This suggests that the controlling factor for the chain transfer reactivity is a perturbation between SOMO of $\dot{\mathbf{C}}$ and LUMO

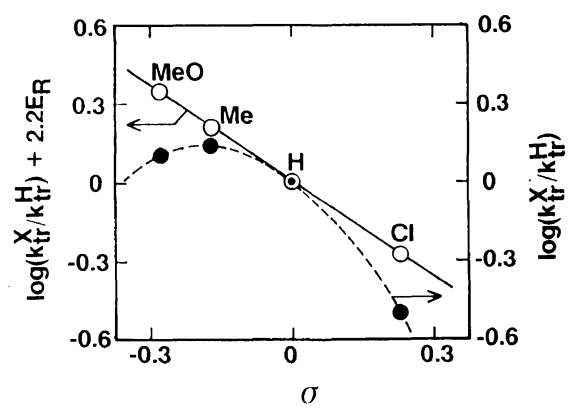

Figure 7. Hammett plot of relative chain transfer rate constant $k_{\mathrm{tr}}^{\mathbf{X}} / k_{\mathrm{tr}}^{\mathrm{H}}$ between $p$-X intermediate carbon radicals and $\mathrm{DPH}$ at $60^{\circ} \mathrm{C}$ in toluene under a nitrogen atmosphere by Hammett's equation $3(\cdots \circ \cdots)$ and modified Hammett's equation $4(-\mathrm{O}-)$. $[\mathrm{X}-\mathrm{EBs}]_{0}=[\mathrm{DPH}]_{0}=1.0$ $\mathrm{moll}^{-1} ;[\mathrm{AIBN}]_{0}=0.04 \mathrm{moll}^{-1} ; \log \left(\mathrm{k}_{\mathrm{tr}}^{\mathrm{X}} / k_{\mathrm{tr}}^{\mathrm{H}}\right)=-1.26 \sigma-$ $2.2 E_{\mathrm{R}}$.

of DPH in the transition state, and the $\dot{\mathbf{C}}$ is a nucleophilic. Furthermore, the negative resonance reaction constant $(\gamma=-2.2)$ as shown in Figure 7 indicates that the resonance effect (R-effect) by the $p$-X substituent on the intermediate carbon radical in the transition state is smaller than that in the initial state. Such an idea with the $\gamma$ value has been discussed by Otsu et al. ${ }^{15,18}$ Consequently, the chain transfer reactivity increases by introducing a large electron-donating group $(+\mathrm{I})$, and decreases by introducing a high resonance stabilizing group $(+\mathrm{R}$ or $-\mathrm{R})$ into the para position of EB.

In the case of polyaddition of PH to DEB, it can be regarded as the addition reaction of PH to EB containing the para ethynyl group. The reactivity of the polyaddition of $\mathrm{PH}$ to DEB is evaluated from the results in Figure 7 to be half reactivity of the addition reaction of PH to EB, assuming $\sigma=0.233,{ }^{19} \gamma=0.11$ (tentative value for $p$-methoxy group ${ }^{15}$ ), and $\rho_{\mathrm{PH}}=\rho_{\mathrm{DPH}}$.

\section{Comparison between $S t^{8}$ and $E B$ in the Addition Reactions of $\mathrm{DPH}$}

DPH added to St and EB without appreciable side reactions to give the adducts of the anti-Markownikoff's structure in $97 \%$ 


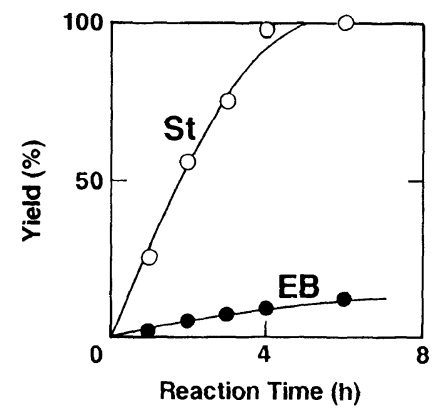

Figure 8. Competitive addition reaction of DPH to St $(\mathrm{O})$ and $\mathrm{EB}(\mathrm{)})$ at $60^{\circ} \mathrm{C}$ in toluene under a nitrogen atmosphere. $[\mathrm{St}]_{0}=[\mathrm{EB}]_{0}=0.5 \mathrm{moll}^{-1} ; \quad[\mathrm{DPH}]_{0}=1.0$ $\mathrm{moll}^{-1} ;[\mathrm{AIBN}]_{0}=0.01 \mathrm{moll}^{-1}$.

yields in both cases. The relative reactivity of St to EB was also determined by a competitive reaction of DPH to $\mathrm{St}$ and $\mathrm{EB}$ with AIBN initiator in toluene at $60^{\circ} \mathrm{C}$ under a nitrogen atmosphere. Figure 8 shows the time-adduct yield curves for the addition reaction of DPH to St and EB. The addition reaction of DPH to $\mathrm{St}$ was completed for $4 \mathrm{~h}$, but the adduct of $\mathrm{DPH}$ and EB was formed at a slow rate as to give a $9 \%$ yield for $4 \mathrm{~h}$. The addition rate of DPH to St was found to be twenty times that of DPH to EB. The rate-determining step of both St and EB systems is the similar chain transfer step, and the transition states of both reactions are also thought to be much alike by the results of the substituent effects (St, $\rho=-0.80 ; \gamma=-1.2 ; \mathrm{EB}, \rho=-1.26 ; \gamma=-2.2$ ). Thus, the difference in the reactivity between St and EB may be estimated by the molecular orbital calculation of the energy gap between SOMO of the each intermediate carbon radical and LUMO of DPH.

Furthermore, based on the above mentioned results, the polyaddition of $\mathrm{PH}$ to $\mathrm{DVB}$ is expected to proceed more rapidly than the polyaddition of $\mathrm{PH}$ to DEB. ${ }^{12}$

\section{Characterization of the Addition Reactions}

The results of a series of the model addition reaction of thiophenol (TP), thiobenzoic acid (TBA), or DPH to St or EB are summarized

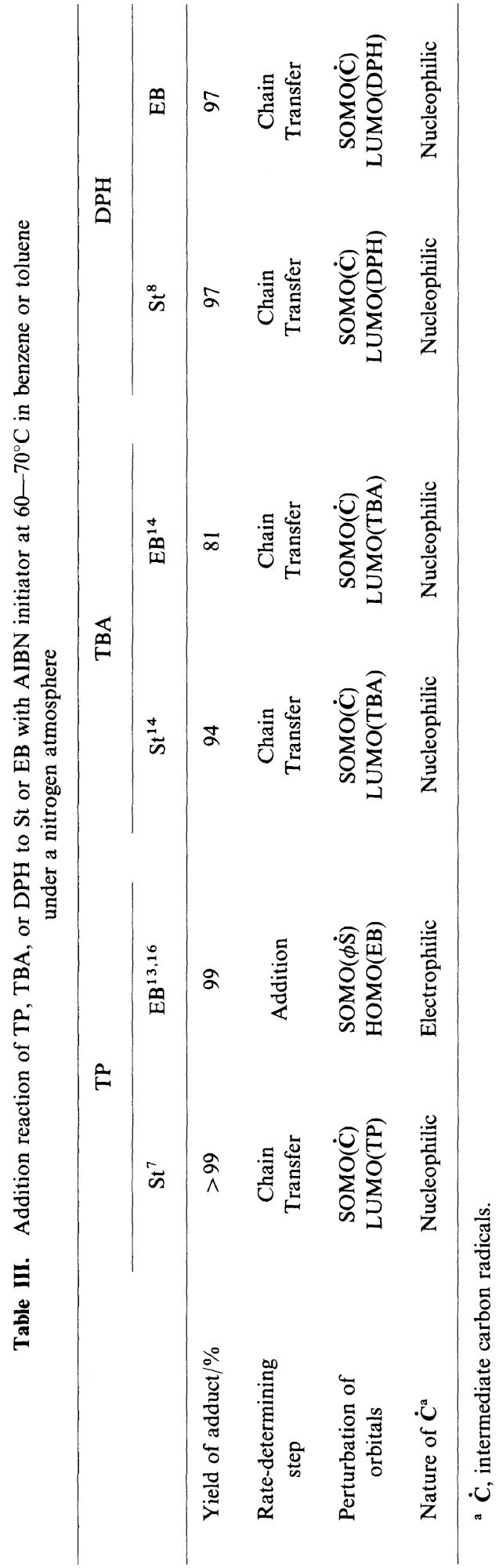

Polym. J., Vol. 25, No. 10, 1993 
in Table III. In all addition reaction except for the reaction between TP and EB, the rate-determining step, the nature of the transition state, and reaction mechanism are much alike, but the reactivity is different. This suggests that the characteristics of these addition reactions are very similar. That is, the controlling factor for the reactivity of these reactions may be energy gap between SOMO of each intermediate carbon, radical and LUMO of related monomers such as TP, TBA, and DPH. In the addition reaction of TP to EB, however, the TP and EB form the CT complex ${ }^{21}$ and the reaction may occur in a cage of the CT complex. That is, the chain transfer step of the reaction may occur very rapidly by a sort of cage effect.

Acknowledgement. The authors are indebted to Mr. K.Tanaka (Idemitsu Kosan Co.) for the measurement of FD mass spectroscopy.

\section{REFERENCES}

1. M. M. Rauhut, H. A. Currier, A. M. Semsel, and V.P.Wystrach, J. Org. Chem., 26, 5138 (1961).

2. G. Maerkl and G. Dannhardt, Tetrahedron Lett., 1455 (1973).

3. R. C. Taylor, R. Kolodny, and D. B. Walters, Synth
Inorg. Metal-Org. Chem., 3, 175 (1973).

4. W. Kuchen and H. Buchwald, Chem. Ber., 95, 2871 (1958).

5. D. J. Dawson, J. D. Frazier, P. J. Brock, and R. J. Twieg, Polym. Mater. Sci. Eng., 55, 401 (1986).

6. A. S. Hay, J. Org. Chem., 25, 637 (1960).

7. E. Kobayashi, T. Obata, S. Aoshima, and J. Furukawa, Polym. J., 22, 803 (1990).

8. T. Obata, E. Kobayashi, S. Aoshima, and J. Furukawa, Polym. J., 25, 1039 (1993).

9. A. G. Christopler and T. R. F. W. Fennell, Microchem. J., 12, 593 (1967).

10. M. Ishihashi and M. Tabushi, Jpn. Anal., 8, 815 (1959).

11. T. Obata, E. Kobayashi, S. Aoshima, and J. Furukawa, to be published in J. Polym. Sci., A, Polym. Chem. Ed.

12. E. Kobayashi, T. Obata, S. Aoshima, and J. Furukawa, Polym. Prepr., Jpn., 40, 1879 (1991).

13. E. Kobayashi, T. Obata, S. Aoshima, and J. Furukawa, Polym. J., 22, 1035 (1990).

14. E. Kobayashi, M. Sakakibara, T. Obata, S. Aoshima, and J. Furukawa, Polym. J., 25, 507 (1993).

15. T. Yamamoto and T. Otsu, J. Soc. Org. Chem. Jpn., 23, 643 (1965).

16. T. Obata, E. Kobayashi, S. Aoshima, and J. Furukawa, Polym. J., 24, 757 (1992).

17. M. Imoto, Kagaku, 29, 958 (1974).

18. T. Otsu, Kogyo Kagaku Zasshi, 70, 1854 (1967).

19. J. A. Landgrebe and R. H. Rynbrandt, J. Org. Chem., 31, 2585 (1966).

20. A. M. Aguiar and T. G. Archibald, Tetrahedron Lett., 5471 (1966).

21. E. Kobayashi, Y. Terada, T. Ohashi, and J. Furukawa, Polym. J., 23, 267 (1991). 